\title{
RESÍDUOS DE SORGO E A MINERALIZAÇÃO DO NITROGÊNIO EM LATOSSOLO VERMELHO FASE CERRADO
}

\author{
Carlos Alberto Vasconcellos ${ }^{1 *}$; Ivanildo Evodio Marriel1'; Fredolino Giacomini dos Santos ${ }^{1}$; Paulo \\ César Magalhães ${ }^{1}$; Cristiane Abreu de Oliveira ${ }^{2}$ \\ ${ }^{1}$ Embrapa Milho e Sorgo, C.P. 151 - CEP: $35701-970$ - Sete Lagoas, MG. \\ ${ }^{2}$ Bolsista FAPEMIG. \\ *Autor correspondente <carlos@cnpms.embrapa.br>
}

\begin{abstract}
RESUMO: As alterações de manejo exercem significativas modificações na qualidade e na quantidade da matéria orgânica, na quantidade e nas formas de $\mathrm{N}$ no solo e no processo de mineralização de $\mathrm{N}$. Neste trabalho, avaliaram-se a taxa de mineralização do $\mathrm{N}$ e a variação de $\mathrm{N}_{-} \mathrm{NH}_{4}^{+}$e $\mathrm{N}-\mathrm{NO}_{3}{ }_{3}^{-}$durante a decomposição dos resíduos de duas cultivares de sorgo. Os tratamentos seguiram o esquema fatorial $2 \times 3 \times 2$, em blocos ao acaso, com dois manejos de solo, três resíduos culturais, dois níveis de nitrogênio e três repetições. Tubos de percolação foram montados com a adição de $20 \mathrm{~g}$ dos solos amostrados, misturados com $20 \mathrm{~g}$ de areia. A cada conjunto de solo mais areia, adicionou-se $0,1 \mathrm{~g}$ do resíduo cultural das cultivares de sorgo, com ou sem $50 \mathrm{mg} \mathrm{kg}^{-1}$ de $\mathrm{N}$, na forma de nitrato de amônio. Após um período de incubação de sete dias, semanalmente

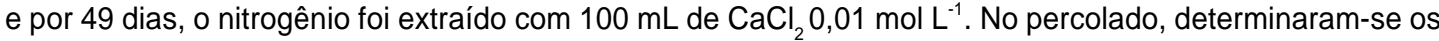

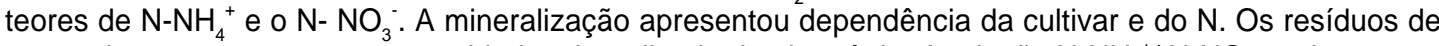
sorgo alteraram o tempo e a quantidade mineralizada de nitrogênio. A relação $\mathrm{N}-\mathrm{NH}_{4}^{+} / \mathrm{N}-\mathrm{NO}_{3}{ }^{-}$variou com o tempo de incubação e com a qualidade do resíduo. Houve aumento até aos quinze dias de incubação e decréscimos nos períodos seguintes. Na ausência de resíduo cultural o solo apresentou maior potencial de mineralização na forma de $\mathrm{N}-\mathrm{NO}_{3}$.

Palavras-chave: amônio, nitrato, cultivar, resíduo vegetal
\end{abstract}

\section{SORGHUM RESIDUES AND NITROGEN MINERALIZATION IN A “CERRADO" OXISOL}

\begin{abstract}
Management alterations induce significant modifications on organic matter quality and quantity, on $\mathrm{N}$ quantity and forms and on the mineralization process. The aim of this work was to study the influence of

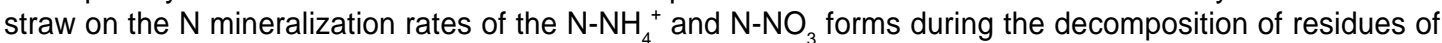
two sorghum genotypes. The experimental design consisted of a $3 \times 2 \times 3$ complete block outline, including two soil managements, three cultural residues and two nitrogen levels, with three replications. Percolation tubes were prepared with $20 \mathrm{~g}$ of soil mixed with $20 \mathrm{~g}$ of sand. Each tube with soil and sand received $0.1 \mathrm{~g}$ residual straw from the two sorghum genotypes, with and without $50 \mathrm{mg} \mathrm{N} \mathrm{kg}^{-1}$ in the form of ammonium nitrate. A control treatment was maintained without straw in the presence and absence of $\mathrm{N}$. After an initial seven day incubation period, $\mathrm{N}$ was extracted weekly, over 49 days, using $100 \mathrm{~mL}$ of $0.01 \mathrm{~mol} \mathrm{~L}^{-1} \mathrm{CaCl}_{2}$. The forms $\mathrm{N}$ -

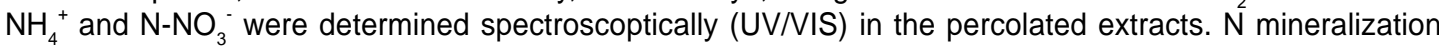
dependent on sorghum genotype and applied $\mathrm{N}$. Sorghum straw influenced time and amount of $\mathrm{N}$ mineralized. The $\mathrm{N}-\mathrm{NH}_{4}{ }^{+} / \mathrm{N}^{-} \mathrm{NO}_{3}$ ratio showed variability during the incubation time as influenced by the incorporated residue. That ratio increased up to fifteen days of incubation time and decreased in subsequent periods. Without residues the mineralization potential was higher in the $\mathrm{N}^{-\mathrm{NO}_{3}}$ form.

Key words: ammonium, nitrate, genotype, plant residue
\end{abstract}

\section{INTRODUÇÃO}

O sorgo, na região central do Brasil, devido à sua rusticidade e pouca necessidade de água, apresenta-se como opção viável para aumentar o rendimento dos produtores. A aplicação de insumos é pequena e não há necessidade de alterações e investimentos no maquinário. Neste caso, o sorgo entra com o aproveitamento das condições residuais deixadas pela soja. Com esse cultivo, há maior exploração do solo por ano agrícola, sendo necessário avaliar as alterações na sua fertilidade, inclusive em função da qualidade do resíduo de sorgo, que poderá influenciar os plantios subsequentes.
Mary et al. (1996) e Vasconcellos et al. (1999) demonstraram a importância do resíduo vegetal na remineralização do $\mathrm{N}$ imobilizado. Campbell et al. (1991), por outro lado, verificaram que a quantidade e a qualidade da matéria orgânica (M.O.) estavam associadas à quantidade de carbono e de nitrogênio que retornava ao solo via resíduo cultural. Esses autores indicaram a taxa inicial de mineralização (TMI) como um índice efetivo em distinguir tanto o valor absoluto como o qualitativo como as alterações que o nitrogênio orgânico sofreu em função das práticas culturais. A TMI foi determinada multiplicando o potencial de mineralização $\left(\mathrm{N}_{0}\right)$ pela constante de mineralização $\left(\mathrm{K}_{\mathrm{o}}\right)$ no tempo zero. Chan et al. (1992) 
verificaram que a redução do carbono orgânico do solo, como resultado de diferentes manejos de solo e cultura, foi acompanhada por significativas reduções no nitrogênio total e nos teores trocáveis de cálcio e de magnésio.

As alterações de manejo do solo e das espécies cultivadas podem, portanto, exercer significativas modificações na qualidade e na quantidade da matéria orgânica, na quantidade e nas formas de $\mathrm{N}$ no solo, principalmente no processo de mineralização de $\mathrm{N}$, na eficiência no aproveitamento de fertilizantes pelas plantas e na movimentação de nutrientes para as camadas mais profundas do solo e alterando a produtividade das culturas.

A qualidade dos resíduos de plantas adicionados ao solo pode, também, alterar a matéria orgânica, influindo na sobrevivência de microrganismos nitrificadores ou amonificadores, em função dessas alterações físicas e químicas. De acordo com Palm \& Sanches (1991), os teores de polifenóis solúveis, expressos como \% de ácido tânico, poderiam explicar a variabilidade da mineralização ou da imobilização do $\mathrm{N}$. No caso, quanto maior os teores de polifenóis, maior seria a magnitude (tempo e quantidade) da imobilização do nitrogênio.

Entre os fatores que influenciam o processo de nitrificação, existem aqueles que controlam as concentrações de $\mathrm{N}-\mathrm{NH}_{4}^{+}, \mathrm{N}-\mathrm{NO}_{2}^{-}$e N-NO${ }_{3}^{-}$no solo. Esses são os inibidores das atividades da urease e dos microorganismos nitrificadores (Victoria et al., 1988). Alguns desses inibidores ocorrem naturalmente, provenientes da liberação de aminoácidos e de bases nitrogenadas liberadas pela decomposição da matéria orgânica do solo ou por substâncias liberadas pelas raízes de algumas plantas (Clark \& Paul, 1970).

É possível que haja comportamento diferenciado de cultivares quanto à interação com a biomassa microbiana no solo, influenciando a relação $\mathrm{N}-\mathrm{NH}_{4}{ }^{+} / \mathrm{N}-\mathrm{NO}_{3}{ }_{3}^{-}$, tanto no seu aspecto qualitativo como quantitativo da nutrição. Também há influência da fonte de nitrogênio sobre a biomassa microbiana do solo, determinando relações $\mathrm{N}-\mathrm{NH}_{4}{ }^{+} / \mathrm{N}-\mathrm{NO}_{3}{ }_{3}^{-}$distintas durante o ciclo da cultura subseqüente à aplicação do resíduo vegetal. A diferença na constituição química do resíduo cultural poderia ocasionar diferentes efeitos na liberação de substâncias inibidoras da nitrificação, tendo-se, por conseqüência, diferenças na relação $\mathrm{N}-\mathrm{NH}_{4}{ }^{+} / \mathrm{N}-\mathrm{NO}_{3}{ }^{-}$em solos tratados com diferentes resíduos.

O objetivo do trabalho foi avaliar a influência dos resíduos de duas cultivares de sorgo na taxa de mineralização do nitrogênio e na variação da suas formas inorgânicas, durante a decomposição desses resíduos, em um LATOSSOLO VERMELHO Distrófico, típico, textura argilosa, fase cerrado, (LVd), cultivado em sistema de plantio direto e convencional.

\section{MATERIAL E MÉTODOS}

O solo (LATOSSOLO VERMELHO Distrófico, típico, textura argilosa, fase cerrado, LVd) foi amostrado, no período outono-inverno de 1997 , na profundidade de 0 a $10 \mathrm{~cm}$, em parcelas experimentais, cujo início dos cultivos iniciaram no período primavera-verão de 1993, com milho e feijão em sucessão. Foram amostradas parcelas com plantio direto e convencional, após o cultivo do milho e imediatamente antes do plantio do feijão, refletindo o tratamento sem adubação nitrogenada em ambas as culturas e com aplicação anual de $8 \mathrm{t} \mathrm{ha}^{-1}$ ano ${ }^{1}$ do resíduo cultural de milho, antes do plantio do feijão que se iniciava após o cultivo do milho. A amostragem dessas áreas foi efetuada com trado tipo holandês, seguindo-se pontos aleatórios, completando-se um volume aproximado de $5 \mathrm{~L}$. A terra foi secada ao ar e uniformizada em granulometria, usando-se peneira com malha de $2 \mathrm{~mm}$. As características químicas desses solos foram: área com plantio convencional $\left(\mathrm{pH}_{\text {água }}=5,3\right.$; $\mathrm{Al}=1,5 \mathrm{mmol}_{\mathrm{c}} \mathrm{dm}^{-3} ; \mathrm{Ca}+\mathrm{Mg}=35,7 \mathrm{mmol}_{\mathrm{c}} \mathrm{dm}^{-3}, \mathrm{~K}=2,5$ $\mathrm{mmol}_{\mathrm{c}} \mathrm{dm}^{-3}, \mathrm{P}_{\text {Mehlich } 1}=13 \mathrm{mg} \mathrm{dm}{ }^{-3}$, M.O. $\left.=37,7 \mathrm{~g} \mathrm{~kg}^{-1}\right)$; área com plantio direto $\left(\mathrm{pH}_{\text {água }}=5,5 ; \mathrm{Al}=3,0 \mathrm{mmol}_{\mathrm{c}} \mathrm{dm}^{-3}\right.$; $\mathrm{Ca}+\mathrm{Mg}=30,9 \mathrm{mmol}_{\mathrm{c}} \mathrm{dm}^{-3}, \mathrm{~K}=2,5 \mathrm{mmol}_{\mathrm{c}} \mathrm{dm}^{-3}, \mathrm{P}_{\text {Mehlich } 1}=38$ $\mathrm{mg} \mathrm{dm}{ }^{-3}$, M.O. $=36,9 \mathrm{~g} \mathrm{~kg}^{1}$ ).

A avaliação do potencial de mineralização do nitrogênio seguiu procedimentos descritos por Stanford \& Smith (1972). Tubos de percolação com capacidade para $250 \mathrm{~mL}$ foram montados com lã de vidro no fundo, para facilitar a obtenção de extratos límpidos. Seguiuse a adição de $20 \mathrm{~g}$ dos solos amostrados, misturados com $20 \mathrm{~g}$ de areia lavada com $\mathrm{HCl} 1 \mathrm{~mol} \mathrm{~L}^{-1}$. A cada conjunto de solo mais areia, adicionou-se $0,1 \mathrm{~g}$ do resíduo cultural moído de duas cultivares de sorgo: BRS $304(\mathrm{C}=$ $41 \%, C: \mathrm{N}=34$ ) e CMS XS 210 A ( $\mathrm{C} \%=40,5 \%, C: N=43)$,

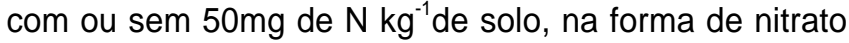
de amônio. Mantiveram-se dois tratamentos-testemunha, sem o resíduo cultural, com ou sem o $\mathrm{N}$. Os tratamentos, portanto, seguiram o esquema fatorial $2 \times 3 \times 2$, sendo dois sistemas de preparo de solo (plantio direto e convencional); três tipos de resíduo cultural (resíduo das duas cultivares de sorgo e solo sem resíduo); dois níveis de $\mathrm{N}$ (0 e $50 \mathrm{mg}$ de $\mathrm{N} \mathrm{kg}^{-1}$ de solo) e três repetições distribuídas em blocos ao acaso. As análises estatísticas, dentro de cada época de amostragem, foram efetuadas através do programa MSTATC, desenvolvido pela Michigan State University (MSTATC, 1991).

Os tubos de percolação foram mantidos em laboratório, cuja temperatura ambiente apresentou amplitude de $15^{\circ} \mathrm{C}$ (temperatura noturna) a $25^{\circ} \mathrm{C}$ (temperatura diurna). Para evitar perdas de umidade, os tubos foram vedados com papel impermeável a água e permeável ao $\mathrm{CO}_{2}$, durante todo o período do ensaio. Ao se iniciar a incubação, o solo recebeu uma quantidade de água suficiente para mantê-lo na capacidade de campo, 0,01 MPa. Após um período inicial de incubação (sete dias), para equilíbrio microbiológico, semanalmente, 
por um período de 49 dias, o nitrogênio foi extraído através da percolação de $100 \mathrm{~mL}$ de $\mathrm{CaCl}_{2} 0,01 \mathrm{~mol} \mathrm{~L}^{-1}$ por unidade experimental. No extrato percolado, determinaram-se os teores de $\mathrm{N}-\mathrm{NH}_{4}^{+}$e $\mathrm{N}-\mathrm{NO}_{3}{ }^{-}$por colorimetria em fluxo contínuo, segundo metodologia descrita por Kamphake et al. (1967) e Alves et al. (1991).

Após cada percolação da solução de cloreto de cálcio, adicionaram-se $50 \mathrm{~mL}$ de solução nutritiva contendo sulfato de potássio $\left(\mathrm{K}_{2} \mathrm{SO}_{4}\right) 0,0025 \mathrm{~mol} \mathrm{~L}^{-1}$; sulfato de magnésio $\left(\mathrm{MgSO}_{4}\right) 0,002 \mathrm{~mol} \mathrm{~L}^{-1}$, sulfato de cálcio $\left(\mathrm{CaSO}_{4} 2 \mathrm{H}_{2} 0\right)$ 0,002 $\mathrm{mol} \mathrm{L}^{-1}$ e $0,005 \mathrm{~mol} \mathrm{~L}^{-1}$ de $\mathrm{Ca}$ $\left(\mathrm{H}_{2} \mathrm{PO}_{4}\right)_{2} . \mathrm{H}_{2} \mathrm{O}$. Após dez minutos, retirou-se 0 excesso dessa solução, fazendo sucção igual a $25 \mathrm{~cm} \mathrm{Hg}$, usando-se um Kitasato para receber o excesso da solução nutritiva após o vácuo.

Os valores de cada extração foram adicionados aos obtidos das extrações anteriores, estabelecendo-se os valores acumulados de cada forma inorgânica de $\mathrm{N}$. Para explicar a variação desses teores, ajustaram-se equações lineares desses valores acumulados, em função da raiz quadrada do tempo de incubação, conforme sugerido por Stanford \& Smith (1972). O potencial de mineralização do nitrogênio foi estimado pela equação:

$$
1 / \mathrm{Nt}=1 / \mathrm{N}_{0}+\mathrm{b} / \mathrm{t}
$$

sendo Nt o nitrogênio mineralizado no tempo determinado; $\mathrm{N}_{0}$ o potencial mineralizável do nitrogênio e t, o tempo (Stanford \& Smith, 1972).

$O T_{1 / 2}$, o tempo necessário para atingir a metade da mineralização, foi calculado através da fórmula:

$$
T_{1 / 2}=0,693 / \mathrm{k}
$$

sendo $\mathrm{k}$ a taxa de mineralização.

O melhor valor de $N_{0}$ foi obtido através de sucessivos ajustamentos de regressões entre o $\left(\mathrm{N}_{0}-\mathrm{Nt}\right)$ e o tempo, através da escolha de diferentes valores de $\mathrm{N}_{\mathrm{o}}$. A declividade da reta obtida entre $0 \ln \left(\mathrm{N}_{\mathrm{o}}-\mathrm{Nt}\right)(\mathrm{y}) \mathrm{e}$ o tempo $(\mathrm{x})$ expressa a taxa de mineralização (Stanford \& Smith, 1972).

Os resultados analíticos foram expressos em $\mathrm{mg}$ de $\mathrm{N} \mathrm{kg}^{-1}$ de solo seco a $105^{\circ} \mathrm{C}$.

\section{RESULTADOS E DISCUSSÃO}

Apesar do histórico de uso de cinco anos em plantio direto, a análise de variância, efetuada dentro de cada tempo de incubação, não indicou influência significativa entre os dois sistemas de preparo de solo nos teores de $\mathrm{N}^{-\mathrm{NO}_{3}}{ }_{3}$ e $\mathrm{N}-\mathrm{NH}_{4}{ }^{+}$, provavelmente, em função da metodologia usada. Portanto, todos os resultados a serem discutidos serão representativos da média dos dois sistemas de preparo. De modo análogo, Mary et al. (1996), ao avaliarem métodos de incorporação de resíduos sobre a mineralização e imobilização de nitrogênio, encontraram diferenças apenas para o tipo de resíduo incorporado.
Nos cultivos convencionais, todo o resíduo é incorporado. A biomassa microbiana do solo possui, portanto, prontamente disponível, a fonte de carbono. No plantio direto, a decomposição dos resíduos é mais lenta e o crescimento dessa biomassa acompanha essa decomposição, através do desenvolvimento de uma flora microbiana mais ativa por maior período de tempo (Follett \& Schimel, 1989). No plantio convencional, a reciclagem é mais rápida, em função do contato do solo com o material vegetal incorporado (Marques et al., 2000).

Principalmente a adição constante de resíduos de milho, com alta relação $\mathrm{C} / \mathrm{N}$, pode ter acarretado uma seleção de microrganismos semelhantes, tanto no plantio direto como no convencional.

O comportamento semelhante entre os dois sistemas de preparo do solo também pode estar associado ao período de amostragem do solo, refletindo o tempo de incorporação dos resíduos de milho dos cultivos anteriores. Nesse caso, os processos de decomposição, mineralização e imobilização podem estar equilibrados imediatamente antes do cultivo do feijão. Há que se considerar, também, o fato de se preparar o solo (secar e peneirar) e acrescentar areia nos tubos de percolação eliminando as possíveis alterações físicas favorecidas pelo plantio direto.

Por outro lado, a metodologia proposta por Stanford \& Smith (1972), ao se efetuar a percolação do $\mathrm{N}$ com cloreto de cálcio, retira-se todo o nitrogênio disponível no solo no momento da percolação, o que deve limitar o desenvolvimento da flora microbiana, apesar da adição de solução nutritiva contendo os demais nutrientes. Essa metodologia, contudo, fornece meios para quantificar possíveis efeitos dos resíduos na mineralização do nitrogênio.

Os valores de $\mathrm{N}^{-\mathrm{NO}_{3}}$ - não se diferenciaram entre os resíduos de sorgo, tanto na presença como na ausência da adição de nitrogênio (TABELA 1); contudo, foram menores quando comparados ao tratamento sem resíduo, demonstrando o efeito do resíduo cultural no processo de imobilizar o nitrogênio. Os valores extraídos de $\mathrm{N}^{-\mathrm{NO}_{3}}$, nos tratamentos sem resíduo, tanto na presença como na ausência de nitrogênio, aumentaram após os 35 dias de incubação. Na presença do resíduo cultural do BRS 304, esse aumento foi verificado apenas na presença de nitrogênio, quando os valores de $\mathrm{N}^{-\mathrm{NO}_{3}}$ aumentaram $141 \%$ entre 35 e 49 dias de incubação.

Os valores de $\mathrm{N}_{-} \mathrm{NH}_{4}^{+}$foram decrescentes com o tempo de incubação e apresentaram diferenças significativas entre os tipos de resíduo cultural apenas aos 21 dias de incubação, sendo obtido maior valor para a BRS $304 \mathrm{com} \mathrm{N}$ e para a CMS XS 210A sem N (TABELA 1).

Os valores acumulados de cada extração permitiram ajustar as equações em função do tempo de incubação (TABELA 2). 
TABELA 1 - Variação das formas de $\mathrm{N}$ extraídas, em função das cultivares de sorgo, adição de $\mathrm{N}$, e da incubação.

\begin{tabular}{|c|c|c|c|c|c|c|c|c|}
\hline \multirow{2}{*}{ Tratamentos ${ }^{1}$} & \multirow{2}{*}{ Tempo } & & \multicolumn{2}{|c|}{$\mathrm{N}-\mathrm{NH}_{4}$} & \multicolumn{2}{|c|}{$\mathrm{N}-\mathrm{NO}_{3}^{-1}$} & \multicolumn{2}{|c|}{$\mathrm{N}-\mathrm{NH}_{4}{ }^{+}+\mathrm{N}-\mathrm{NO}_{3}^{-1}$} \\
\hline & & & Com N & Sem N & Com N & Sem N & Com N & Sem N \\
\hline & dias & & ------------------- & ------------ & --------- mg & $\mathrm{kg}^{-1}--\cdots$ & - & - \\
\hline BRS 304 & 7 & & $39 \mathrm{Aa}$ & $5,4 \mathrm{Ab}$ & $51,4 \mathrm{Aa}$ & $9,3 \mathrm{Ab}$ & $90,4 \mathrm{Aa}$ & $14,7 \mathrm{Ab}$ \\
\hline CMS XS210A & & & $39,5 \mathrm{Aa}$ & $6,3 \mathrm{Ab}$ & $57,4 \mathrm{Aa}$ & $7,9 \mathrm{Ab}$ & $96,9 \mathrm{Aa}$ & $14,2 \mathrm{Ab}$ \\
\hline \multirow[t]{2}{*}{ Testemunha } & & & $40 \mathrm{Aa}$ & $5,9 \mathrm{Ab}$ & $56,4 \mathrm{Aa}$ & $9,5 \mathrm{Ab}$ & $96,4 \mathrm{Aa}$ & $15,4 \mathrm{Ab}$ \\
\hline & & CV\% & 24 & & 18 & & 19 & \\
\hline BRS 304 & 14 & & $2,0 \mathrm{Aa}$ & $1,8 \mathrm{Aa}$ & $0,52 \mathrm{Ba}$ & $0,52 \mathrm{Ba}$ & $2,5 \mathrm{Ba}$ & 2,3 Ba \\
\hline CMS XS210A & & & $1,5 \mathrm{Aa}$ & $1,7 \mathrm{Aa}$ & $0,52 \mathrm{Ba}$ & $0,52 \mathrm{Ba}$ & $2,0 \mathrm{Ba}$ & 2,2 Ba \\
\hline \multirow[t]{2}{*}{ Testemunha } & & & 2,2 Aa & 2,0 Aa & $6,1 \quad \mathrm{Aa}$ & $3,9 \quad \mathrm{Ab}$ & $8,3 \mathrm{Aa}$ & $5,9 \mathrm{Aa}$ \\
\hline & & CV\% & 40 & & 140 & & 73 & \\
\hline BRS 304 & 21 & & $1,5 \mathrm{Aa}$ & $0,8 \mathrm{Bb}$ & $0,52 \mathrm{Ba}$ & $0,52 \mathrm{Ba}$ & $2,0 \mathrm{ABa}$ & 1,3 Bb \\
\hline CMS XS210A & & & $1,0 \mathrm{Ba}$ & $0,9 \mathrm{Aba}$ & $0,52 \mathrm{Ba}$ & $0,52 \mathrm{Ba}$ & $1,5 \mathrm{Ba}$ & 1,5 Ba \\
\hline \multirow[t]{2}{*}{ Testemunha } & & & $1,1 \mathrm{Ba}$ & $1,1 \mathrm{Aa}$ & $1,1 \quad \mathrm{Aa}$ & $1,2 \mathrm{Aa}$ & 2,2 Aa & 2,3 $\mathrm{Aa}$ \\
\hline & & CV\% & 21 & & 19 & & 35 & \\
\hline BRS 304 & 28 & & $1,4 \mathrm{Aa}$ & $0,6 \mathrm{Ab}$ & $0,52 \mathrm{Ba}$ & $0,52 \mathrm{Ba}$ & 1,9 Aa & $1,1 \mathrm{Ab}$ \\
\hline CMS XS210A & & & $0,9 \mathrm{Aa}$ & $0,6 \mathrm{Aa}$ & $0,52 \mathrm{Ba}$ & $0,52 \mathrm{Ba}$ & $1,4 \mathrm{Ba}$ & $1,1 \mathrm{Aa}$ \\
\hline \multirow[t]{2}{*}{ Testemunha } & & & $1,1 \mathrm{Aa}$ & $0,6 \mathrm{Ab}$ & $0,91 \mathrm{Aa}$ & $0,63 \mathrm{Ab}$ & $2,0 \mathrm{Aa}$ & $1,3 \mathrm{Ab}$ \\
\hline & & CV\% & 40 & & 31 & & 23 & \\
\hline BRS 304 & 35 & & $0,82 \mathrm{Aa}$ & $0,55 \mathrm{Ab}$ & $0,54 \mathrm{Bb}$ & $0,56 \mathrm{Bb}$ & $1,4 \mathrm{Ba}$ & 1,1 Ba \\
\hline CMS XS210A & & & $0,79 \mathrm{Aa}$ & $0,55 \mathrm{Ab}$ & $0,58 \mathrm{Bb}$ & $0,61 \mathrm{Bb}$ & $1,4 \mathrm{Ba}$ & $1,2 \mathrm{Ba}$ \\
\hline \multirow[t]{2}{*}{ Testemunha } & & & $1,0 \quad \mathrm{Aa}$ & $0,65 \mathrm{Ab}$ & $2,3 \quad \mathrm{Aa}$ & $1,4 \quad A b$ & 3,3 Aa & 2,0 Aa \\
\hline & & CV\% & 34 & & 69 & & 47 & \\
\hline BRS 304 & 42 & & $0,59 \mathrm{Ab}$ & $0,85 \mathrm{Aa}$ & $1,1 \quad \mathrm{Ba}$ & $0,59 \mathrm{Ba}$ & 1,7 Ba & $1,5 \mathrm{Aa}$ \\
\hline CMS XS210A & & & $0,63 \mathrm{Ab}$ & $1,02 \mathrm{Ab}$ & $0,91 \mathrm{Ba}$ & $1,26 \mathrm{ABa}$ & $1,5 \mathrm{Ba}$ & 2,3 ABa \\
\hline \multirow[t]{2}{*}{ Testemunha } & & & $0,88 \mathrm{Aa}$ & $0,95 \mathrm{Aa}$ & $2,14 \mathrm{Aa}$ & $1,67 \mathrm{Aa}$ & $3,0 \mathrm{Aa}$ & 2,6 Aa \\
\hline & & CV\% & 42 & & 80 & & 51 & \\
\hline BRS 304 & 49 & & $0,31 \mathrm{Aa}$ & $0,35 \mathrm{Aa}$ & $1,3 \mathrm{Aa}$ & $0,6 \mathrm{Aa}$ & $1,6 \mathrm{Aa}$ & $0,9 \mathrm{Aa}$ \\
\hline CMS XS210A & & & $0,31 \mathrm{Aa}$ & $0,31 \mathrm{Aa}$ & $1,0 \mathrm{Aa}$ & $0,9 \mathrm{Aa}$ & 1,3 Aa & $1,2 \mathrm{Aa}$ \\
\hline \multirow[t]{2}{*}{ Testemunha } & & & $0,31 \mathrm{Aa}$ & $0,28 \mathrm{Aa}$ & $1,8 \mathrm{Aa}$ & $1,4 \mathrm{Aa}$ & $2,1 \mathrm{Aa}$ & $1,7 \mathrm{Aa}$ \\
\hline & & CV\% & 34 & & 73 & & 58 & \\
\hline
\end{tabular}

${ }^{1}$ Médias seguidas pela mesma letra não apresentam diferenças significativas, pelo teste de Tukey, a 5\%. Letras maiúsculas comparam resíduos; letras minúsculas, níveis de $\mathrm{N}$.

Os coeficientes angulares dessas equações indicaram que, na ausência do nitrogênio e na presença do resíduo cultural, houve menores liberações de $\mathrm{N}(\mathrm{N}$ $\mathrm{NO}_{3}{ }^{-}$e $\mathrm{N}-\mathrm{NO}_{3}{ }^{-}+\mathrm{NH}_{4}{ }^{+}$), quando comparadas como o tratamento sem resíduo, indicando o efeito do resíduo com alta relação $\mathrm{C}: \mathrm{N}$ na rápida imobilização do nitrogênio (Mengel, 1996).

Para os valores de $\mathrm{N}-\mathrm{NH}_{4}{ }^{+}$estes coeficientes demonstraram que, na presença do nitrogênio, aumentaram mais na presença dos resíduos da cultivar BRS 304. Sendo a forma de $\mathrm{N}_{-} \mathrm{NH}_{4}^{+}$a predominante nos estádios iniciais da mineralização, pode-se inferir que houve re- mineralização do $\mathrm{N}$, estando de acordo com sua menor relação C:N (34), em comparação ao CMS XS 210 A, com maior relação C:N (43) e menor coeficiente angular. Pode-se, também, sugerir a possibilidade do efeito diferencial dos resíduos sobre o processo de mineralização, particularmente os teores de lignina e polifenóis (Palm \& Sanches, 1991).

Apesar de esses resultados terem sido obtidos em condições de laboratório, na ausência de plantas, suas conseqüências refletem a alteração na relação $\mathrm{N}$ $\mathrm{NH}_{4}^{+} / \mathrm{N}^{-\mathrm{NO}_{3}}$. Quando há predomínio de uma forma sobre a outra, há alterações na proporção cátions/ânions absorvidos, podendo alterar a eficiência nutricional e a produtividade das culturas (Gashaw \& Mugwira, 1981; Lamaze et al., 1984; Vale et al., 1998). 
TABELA 2 - Coeficientes angular $\left(b_{1}\right)$ e valores da interseção (a) das equações ajustadas do total acumulado de nitrogênio mineralizado $(\mathrm{y})$ em função da raiz quadrada do tempo $(\mathrm{x})$.

\begin{tabular}{|c|c|c|c|c|c|c|}
\hline \multirow{3}{*}{ Tratamentos } & \multicolumn{6}{|c|}{ Coeficientes } \\
\hline & \multicolumn{3}{|c|}{$b_{1}$} & \multicolumn{3}{|c|}{$\mathrm{a}$} \\
\hline & $\mathrm{N}-\mathrm{NH}_{4}{ }^{+}$ & $\mathrm{N}-\mathrm{NO}_{3}^{-}$ & $\mathrm{N}-\mathrm{NH}_{4}{ }^{+}+\mathrm{NO}_{3}^{-}$ & $\mathrm{N}-\mathrm{NH}_{4}^{+}$ & $\mathrm{N}-\mathrm{NO}_{3}^{-}$ & $\mathrm{N}-\mathrm{NH}_{4}^{+}+\mathrm{NO}_{3}$ \\
\hline & \multicolumn{6}{|c|}{ Com Nitrogênio } \\
\hline \multirow[t]{2}{*}{ BRS 304} & $1,81 \mathrm{a}$ & $0,95 \mathrm{~b}$ & $2,79 \mathrm{~b}$ & $33,96 \mathrm{~b}$ & $48,41 \mathrm{~b}$ & $82,11 b$ \\
\hline & $(99 \%)$ & $(90 \%)$ & $(98 \%)$ & & & \\
\hline \multirow[t]{2}{*}{ CMS XS 210A } & $1,21 \mathrm{c}$ & $0,89 \mathrm{~b}$ & $2,10 \mathrm{c}$ & $36,44 \mathrm{a}$ & $54,65 \mathrm{a}$ & $90,85 \mathrm{a}$ \\
\hline & $(99 \%)$ & $(93 \%)$ & $(99 \%)$ & & & \\
\hline \multirow[t]{3}{*}{ TESTEMUNHA } & $1,50 \mathrm{~b}$ & $3,00 \mathrm{a}$ & $4,47 \mathrm{a}$ & $36,33 \mathrm{a}$ & $49,61 \mathrm{~b}$ & $85,73 b$ \\
\hline & $(99 \%)$ & $(95 \%)$ & $(99 \%)$ & & & \\
\hline & \multicolumn{6}{|c|}{ Sem Nitrogênio } \\
\hline \multirow[t]{2}{*}{ BRS 304} & $1,15 \mathrm{a}$ & $0,76 \mathrm{~b}$ & $1,85 \mathrm{~b}$ & $2,45 c$ & $7,03 \mathrm{a}$ & $9,85 \mathrm{a}$ \\
\hline & $(97 \%)$ & $(97 \%)$ & $(99 \%)$ & & & \\
\hline \multirow[t]{2}{*}{ CMS XS 210A } & $1,12 \mathrm{a}$ & $0,95 \mathrm{~b}$ & $2,10 \mathrm{~b}$ & $3,56 \mathrm{a}$ & $4,89 \mathrm{~b}$ & $8,35 \mathrm{~b}$ \\
\hline & $(98 \%)$ & $(91 \%)$ & $(98 \%)$ & & & \\
\hline \multirow[t]{2}{*}{ TESTEMUNHA } & $1,26 a$ & $2,11 \mathrm{a}$ & $3,37 \mathrm{a}$ & $2,92 \mathrm{~b}$ & $4,55 \mathrm{~b}$ & $7,46 \mathrm{c}$ \\
\hline & $(98 \%)$ & $(97 \%)$ & $(98 \%)$ & & & \\
\hline
\end{tabular}

${ }^{1}$ Médias seguidas pela mesma letra, nas colunas, não diferem pelo teste dos coeficientes de regressão a $5 \%$. O número entre parênteses refere-se aos coeficientes de determinação significativos a $1 \%$.

$\mathrm{Na}$ ausência da adição do nitrogênio houve

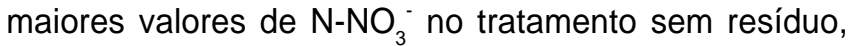
demonstrando o efeito do resíduo em diminuir o N-NO${ }_{3}$. no solo, provavelmente, associado ao desenvolvimento da microbiota do solo. Green et al. (1995) mencionaram que, em solo com pH 5,8 e com a incorporação de resíduos de milho, a presença do nitrato diminuiu a decomposição da matéria orgânica do solo e aumentou a decomposição do resíduo cultural. A presença do nitrato, portanto, poderia ser um indicador de sustentabilidade do sistema, ao refletir menor taxa de decomposição da matéria orgânica.

$\mathrm{Na}$ Figura 1, estão os valores obtidos para a relação $\mathrm{N}-\mathrm{NH}_{4}^{+} / \mathrm{N}-\mathrm{NO}_{3}{ }_{3}$, em cada extração efetuada durante o período de incubação. Esses valores, em função do processo extrativo de todas as formas de $\mathrm{N}$ existentes no período determinado, podem não refletir as condições de campo, onde há uma absorção contínua, crescente e preferencial com o desenvolvimento da planta e com a cultivar. Todavia, reflete a influência do tipo de resíduo cultural, principalmente na presença de nitrogênio, nas formas de nitrogênio durante a sua decomposição. $\mathrm{Na}$ ausência de $\mathrm{N}$ não houve diferenças estatísticas entre os tratamentos, provavelmente em função de menor desenvolvimento da biomassa microbiana.

A taxa de mineralização (TABELA 3) alterou-se, principalmente em função da adição de nitrogênio sendo maior na ausência dos resíduos culturais de sorgo. 0 solo apresentou maior potencial de mineralização de $\mathrm{N}$ na forma $\mathrm{N}-\mathrm{NO}_{3}$.

\section{$\operatorname{COMN}$}

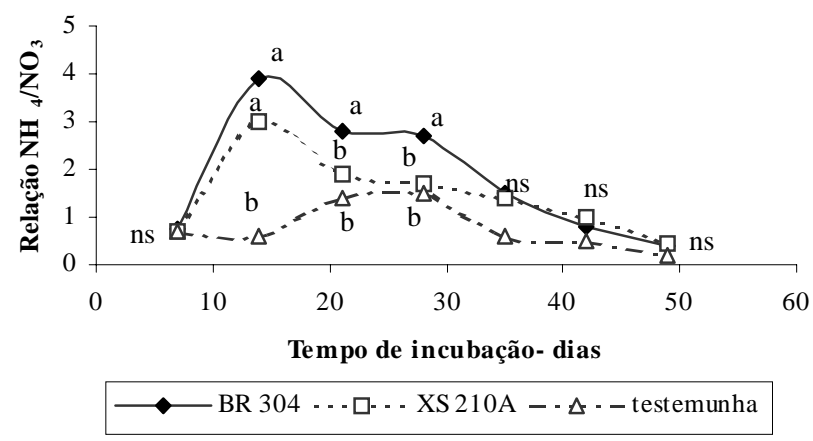

SEM N

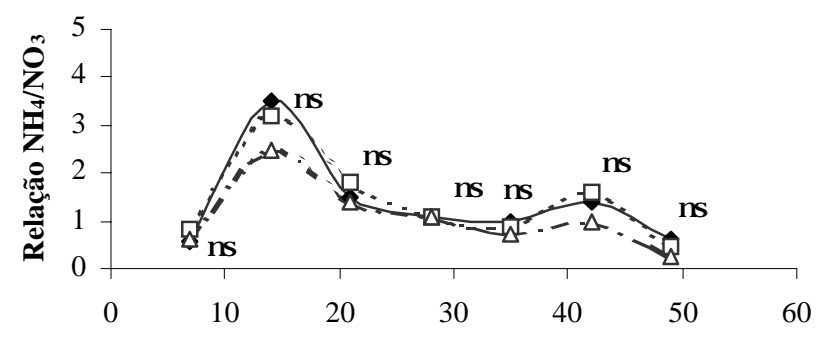

Tempo de incubação-dias

Figura 1 - Variação da relação $\mathrm{N}-\mathrm{NH}^{+}{ }_{4} / \mathrm{N}^{-} \mathrm{NO}_{3}-$ em função dos tipos de resíduo e do tempo de incubação em solo LVd. Médias seguidas pela mesma letra não apresentam diferenças significativas, entre resíduos, dentro de uma mesma época, pelo teste de Tukey a $5 \%$. 
Os valores de $T_{1 / 2}, 0$ tempo (em dias) no qual a metade do $\mathrm{N}_{0}$ é mineralizada, foram sempre menores na presença de resíduo e do $\mathrm{N}$ em relação aos tratamentos sem resíduo cultural, exceto para a forma de $\mathrm{N}_{-} \mathrm{NO}_{3}$ quando se incorporaram resíduos da cultivar CMSXS 210.

$\mathrm{Na}$ presença de $50 \mathrm{mg} \mathrm{kg}^{-1}$ de nitrogênio, no tratamento sem resíduo, houve aumento de $83 \mathrm{mg}$ de $\mathrm{N}\left(\mathrm{NH}_{4}^{+} \mathrm{NO}_{3}{ }^{-}\right) \mathrm{kg}^{-1}$ de solo, indicando o estímulo do nitrogênio na biomassa microbiana em aumentar o $\mathrm{N}$ mineralizável. Esse aumento foi de $34 \mathrm{mg} \mathrm{kg}^{-1}$ para a forma de $N-\mathrm{NH}_{4}^{+}$e de $49 \mathrm{mg} \mathrm{kg}^{-1}$ para a forma de $\mathrm{N}$ $\mathrm{NO}_{3}^{-}$(TABELA 3).

TABELA 3 - Estimativas do potencial mineralizável, $\mathrm{N}_{\circ}$, taxa de mineralização, $k$ e tempo para atingir metade da mineralização, $T_{1 / 2}$, em tratamentos com diferentes resíduos e adição de nitrogênio.

\begin{tabular}{|c|c|c|c|}
\hline \multirow{2}{*}{ Tratamentos } & \multicolumn{3}{|c|}{ Parâmetros } \\
\hline & $\mathrm{N}_{0}\left(\mathrm{mg} \mathrm{kg}^{-1}\right)$ & $\mathrm{K}\left(\operatorname{dias}^{-1}\right)$ & $T_{1 / 2}\left(\right.$ dias s$\left.^{-1}\right)$ \\
\hline & \multicolumn{3}{|c|}{$\mathrm{N}-\mathrm{NH}_{4}^{+-}$} \\
\hline & \multicolumn{3}{|c|}{ Com Nitrogênio } \\
\hline BRS 304 & 46,5 & 0,068 & 10,1 \\
\hline $\begin{array}{l}\text { CMS XS } \\
210 A\end{array}$ & 44,8 & 0,082 & 8,4 \\
\hline \multirow[t]{2}{*}{ Sem resíduo } & 46,9 & 0,065 & 10,7 \\
\hline & \multicolumn{3}{|c|}{ Sem Nitrog ênio } \\
\hline BRS 304 & 11,9 & 0,033 & 21,1 \\
\hline $\begin{array}{l}\text { CMS XS } \\
210 A\end{array}$ & 12,2 & 0,045 & 15,5 \\
\hline \multirow[t]{3}{*}{ Testemunha } & 12,9 & 0,037 & 18,7 \\
\hline & \multicolumn{3}{|c|}{$\mathrm{N}-\mathrm{NO}_{3}$} \\
\hline & \multicolumn{3}{|c|}{ Com Nitrog ênio } \\
\hline BRS 304 & 54,6 & 0,090 & 7,7 \\
\hline $\begin{array}{l}\text { CMS XS } \\
210 A\end{array}$ & 60,6 & 0,082 & 8,5 \\
\hline \multirow[t]{2}{*}{ Sem Resíduo } & 70,9 & 0,113 & 6,1 \\
\hline & \multicolumn{3}{|c|}{ Sem Nitrogênio } \\
\hline BRS 304 & 12,2 & 0,068 & 10,2 \\
\hline $\begin{array}{l}\text { CMS XS } \\
210 A\end{array}$ & 11,3 & 0,097 & 7,1 \\
\hline \multirow[t]{3}{*}{ Testemunha } & 21,2 & 0,042 & 16,4 \\
\hline & \multicolumn{3}{|c|}{$\mathrm{N}-\mathrm{NH}_{4}^{+}{ }_{4} \mathrm{~N}-\mathrm{NO}_{3}{ }^{-}$} \\
\hline & \multicolumn{3}{|c|}{ Com Nitrog ênio } \\
\hline BRS 304 & 101,2 & 0,086 & 8,1 \\
\hline $\begin{array}{l}\text { CMS XS } \\
210 A\end{array}$ & 105,5 & 0,063 & 11,0 \\
\hline \multirow[t]{2}{*}{ Sem Resíduo } & 117,9 & 0,068 & 10,1 \\
\hline & \multicolumn{3}{|c|}{ Sem Nitrog ênio } \\
\hline BRS 304 & 24,1 & 0,071 & 9,8 \\
\hline $\begin{array}{l}\text { CMS XS } \\
210 A\end{array}$ & 23,5 & 0,054 & 12,8 \\
\hline Testemunha & 34,2 & 0,03 & 18,2 \\
\hline
\end{tabular}

Scientia Agricola, v.58, n.2, p.373-379, abr./jun. 2001
Quando houve a incorporação do resíduo, o valor de $\mathrm{N}_{0}$ diminuiu, dentro do período de incubação em estudo. A diferença entre o total de $\mathrm{N}\left(\mathrm{NH}_{4}{ }^{+}+\mathrm{NO}_{3}{ }^{-}\right)$ não demonstrou alterações no potencial de $\mathrm{N}$ mineralizável, entre as cultivares de sorgo. Tanto na presença como na ausência de $\mathrm{N}$, esse potencial decresceu ao redor de $13 \mathrm{mg} \mathrm{kg}^{-1}$. A forma de $\mathrm{N}^{-\mathrm{NH}_{4}^{+}}$ não se alterou, quando comparada com o tratamento sem resíduo; contudo, a forma de $\mathrm{N}^{-\mathrm{NO}_{3}}{ }_{3}^{-}$decresceu aproximadamente $10 \mathrm{mg} \mathrm{kg}^{-1}$.

$\mathrm{O}$ consumo de $\mathrm{N}^{-\mathrm{NO}_{3}}{ }_{3}^{-}$inclui a imobilização pela biomassa microbiana, redução à $\mathrm{N}^{-} \mathrm{NH}_{4}^{+}$e a desnitrificação. Como não houve excesso de umidade, pode-se afirmar que o consumo de $\mathrm{N}^{-\mathrm{NO}_{3}}{ }_{3}^{-}$é igual ao imobilizado durante o período de incubação.

\section{CONCLUSÕES}

A incorporação de resíduos culturais de sorgo alteram o tempo e a quantidade de nitrogênio mineralizada.

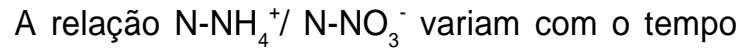
de incubação e com o tipo do resíduo cultural incorporado.

$\mathrm{Na}$ ausência de resíduo cultural o solo apresentou maior potencial de mineralização na forma de N-NO${ }_{3}$.

\section{REFERÊNCIAS BIBLIOGRÁFICAS}

ALVES, B. J. R.; SOUTO C.M.; FERREIRA, E.; URQUIAGA, S.; BODDEY, R.M. Avaliação da sensibilidade de três métodos

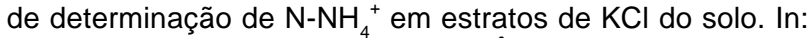
CONGRESSO BRASILEIRO DE CIÊNCIA DO SOLO, 23., Porto Alegre, 1991. Programas e resumos. Porto Alegre: SBCS, 1991. p.190.

CAMPBELL, C.A.; LAFOND, G.P.; LEYSHON, A.J.; ZENTNER, R.P.; JANZEN, H.H. Effect of cropping practices on the initial potential rate of $\mathrm{N}$ mineralization in thin Black Chernozem. Canadian Journal of Soil Science, v.71, p.43-53 , 1991.

CHAN , K.Y.; ROBERTS, W.P.; HEENAN, W.P. Organic carbon and associated soil properties of a Red Earth after 10 years of rotation under different stubble and tillage practices. Australian Journal of Research, v.30, p.71-83, 1992.

CLARK, F.E.; PAUL, E.A. The microflora of grassland. Advances in Agronomy, v.22, p.375-435, 1970.

FOLLETT,R.F.; SCHIMEL, D.S. Effects of tillage practices on microbial biomass dynamics. Soil Science Society of America Journal, v.53, p.1091-1096, 1989.

GASHAW, I.; MUGWIRA, L.M. Ammonium-N and nitrate-N effects on the growth and mineral compositions of triticale, wheat and rye. Agronomy Journal, v.73, p.47-51, 1981.

GREEN, C.J.; BLACKMER, A.M.; HORTON, R. Nitrogen effects on conservation of carbon during corn residue decomposition in soil. Soil Science Society of America Journal, v.59, p.453459, 1995.

KAMPHAKE, L.J.; HANNAH, S.A.; COHEN, J.M. Automated analysis for nitrate by hidrazine reduction. Water Research, v.7, p.205-216, 1967.

LAMAZE, T.; SENTENAC, H.; GRIGNON, C. Effects of nitrate on phosphate accumulation and transport by corn roots. Physiologie Vegetale, v.22, p.155-161, 1984. 
MARQUES, T.C.L.L. S; VASCONCELLOS, C.A.; PEREIRA FILHO, I.; FRANÇA, G.E.de; CRUZ, J.C. Evolvimento de dióxido de carbono e mineralização de nitrogênio em Latossolo Veremelho-Escuro com diferentes manejos. Pesquisa Agropecuária Brasileira, v.35, p.581-589, 2000.

MARY, B.; RECOUS, S.; DARWIS, D.; ROBIN, D. Interactions between decomposition of plant residues and nitrogen cycling in soil. Plant and Soil, v.181, p.71-82, 1996.

MENGEL, K. Turnover of organic nitrogen in soils and its availability to crops. Plant and Soil, v.181, p.83-93, 1996.

MSTAT-C. Michigan State University: Crop and Science Department, 1991.

PALM, C.A.; SANCHES, P.A. Nitrogen release from the leaves of some tropical legumes as affected by their lignin and polyphenolic contents. Soil Biology and Biochemistry, v.23, p.83-88, 1991.
STANFORD, G.S.; SMITH, S.J. Nitrogen mineralization potentials of soils. Soil Science Society of America Proceedings, v.36, p.465-472, 1972.

VALE, F.R.; GUAZELLI, E.M.F.; FURTINI NETO, A.E. ; FERNANDES, L.A. Cultivo do feijoeiro em solução nutritiva sob proporções variáveis de amônio e nitrato. Revista Brasileira de Ciência do Solo, v.22, p.35-42, 1998.

VASCONCELLOS, C.A.; CAMPOLINA, D.C.A.; SANTOS, F.G.; PITTA, G.V.E.; MARRIEL, I.E. Resposta da soja e da biomassa de carbono do solo aos resíduos de cinco genótipos de sorgo. Revista Brasileira de Ciência do Solo, v.23, p. 69-77, 1999.

VICTORIA, R.L.; COLAÇO, W.; MAGALHÃES, A.M.T. Mineralização e volatilização de nitrogênio do solo. In: CONGRESSO BRASILEIRO DE CIÊNCIA DO SOLO, 21., Guarapari, 1987. Anais. Campinas: SBCS, 1988. p.379-387.

Recebido em 11.01 .00 